\title{
A Note on Ball Proximinality
}

\author{
Bo Wang ${ }^{*}$, Xiaoyan Liu ${ }^{2}$ \\ ${ }^{1}$ School of Mathematical Sciences, Huaqiao University, Quanzhou, China \\ ${ }^{2}$ Chengyi University College, Jimei University, Xiamen, China \\ Email: ^wangbo2013@hqu.edu.cn, liuxiaoyan@jmu.edu.cn
}

How to cite this paper: Wang, B. and Liu, X.Y. (2020) A Note on Ball Proximinality. Applied Mathematics, 11, 1196-1203. https://doi.org/10.4236/am.2020.1111081

Received: October 28, 2020

Accepted: November 22, 2020

Published: November 25, 2020

Copyright $\odot 2020$ by author(s) and Scientific Research Publishing Inc. This work is licensed under the Creative Commons Attribution International License (CC BY 4.0)

http://creativecommons.org/licenses/by/4.0/

\begin{abstract}
In this paper, we prove when these $x \in l_{2}$ with $d\left(x, B_{l_{2}}\right)>\frac{1}{12}$, they have the common $\delta$ for strongly ball proximinal. By using this property, we can prove the strong ball proximinality of $l_{\infty}\left(l_{2}\right)$. Also, we show that equable subspace $Y$ of a Banach space $X$ is actually uniform ball proximinality.
\end{abstract}

\section{Keywords}

Ball Proximinal, Strongly Ball Proximinal, Uniformly Ball Proximinal, Equable Spaces

\section{Introduction}

The best approximation is one of the most important concepts in approximation theory, and it plays an important role in many scientific fields. For example, it has full application in Banach space geometry theory, smooth analysis, function approximation, optimization theory and other disciplines. Many researchers have conducted a lot of in-depth study on the proximinal set (especially the proximinal subspace) [1]-[8]. When people focus on the proximinal subspace, they find the (strong) proximinlality of unit ball $B_{Y}$ of a subspace $Y$ is stronger than the (strong) proximinality in $Y$. In [9], Saidi showed for any nonreflexive space $Y$ there is a Banach space $X$ such that $Y$ is isometrically isomorphic to a subspace $Z$ of $X$ such that $Z$ is proximinal in $X$ but not ball proximinal in $X$. ([10], Example 3.3) showed that the space $Z$ is strongly proximinal in $X$. Based on these results, the notion of ball proximinality, which focuses the problem of best approximation from linear subspaces on non-linear convex sets was introduced in 2007 in the paper [10] by Bandyopadhyay et al. Later, this new concept has been extensively studied [11]-[17]. To characterize ball proximinal and strongly ball proximinal hyperplanes, Indumathi and Prakash [11] introduced 
the so called $E$-proximinality. Then Lin et al. [12] generalize those results from $E$-proximinal hyperplanes to $E$-proximinal subspaces. Lalithambigai [13] study the ball proximinality of equable spaces and prove an equable subspace is strongly ball proximinal.

In general, there are a few results about stability of the ball proximinality. Firstly, Bandyopadhyay et al. [10] showed if $\left(X_{n}\right)$ and $\left(Y_{n}\right)$ are two sequences of Banach spaces such that $Y_{n}$ is a subspace of $X_{n}$ that is ball proximinal in $X_{n}$ for each $n$, then the $c_{0}$-direct sum $\left(\sum \oplus Y_{n}\right)_{c_{0}}$ is ball proximinal in $\left(\sum \oplus X_{n}\right)_{c_{0}}$. Paul [14] showed stability of ball proximinality and strongly ball proximinality in spaces of Bochner integrable functions. Then, fruitful results about ball proximinality and strong ball proximinality were obtained in [15]. For example, it has been proved if $E$ is a Banach space with a uniformly monotone 1-unconditional basis (e.g. $E=l_{p}$ for $\left.1 \leq p<\infty\right)$ or $E$ is $c_{0}$, then $\left(\sum \oplus Y_{n}\right)_{E}$ is strongly ball proximinal in $\left(\sum \oplus X_{n}\right)_{E}$, where $Y_{n}$ is a subspace of $X_{n}$ that is strongly ball proximinal in $X_{n}$ for each $n$.

For $E=l_{\infty}$, it seems difficult to get a general answer to the stability of strong ball proximinality. So it is possible to consider some special cases as $l_{\infty}(X)$ and to find the proper conditions for a Banach space $X$ such that the unit ball of $l_{\infty}(X)$ is strongly proximinal. In this paper, we can see for $X=l_{2}, l_{\infty}(X)$ is strongly proximinal because for these $x \in l_{2}$ with $d\left(x, B_{l_{2}}\right)>\frac{1}{12}$, they have the common $\delta$ for strong ball proximinality, then we can get the strong ball proximinality of $l_{\infty}\left(l_{2}\right)$. Paul [14] developed the notion of "uniform proximinality" of a closed convex set in a Banach space and gave some examples to have this property. Also, we can give another example. That is motivated by the proof in [13], we show that equable subspace $Y$ of a Banach space $X$ is uniform ball proximinality.

\section{Preliminaries}

We will now present the notations and definitions that would be used throughout the paper. Let $X$ denotes a real Banach space. Also, we assume that all subspaces are closed. The closed unit ball of $X$ is denoted by $B_{X}$ and $B_{X}=\{x \in X:\|x\| \leq 1\}$. For $x \in X$ and $r>0$, we set $B(x, r)=\{y \in X:\|y-x\| \leq r\}$.

Let $C$ be a nonempty closed convex subset of $X$. For any $x \in X$ and $\delta>0$, $P_{C}(x)$ denote the sets:

$$
P_{C}(x)=\{y \in C:\|x-y\|=d(x, C)\},
$$

where $d(x, C)$ is the distance of $x$ to $C$, that is $d(x, C)=\inf \{\|x-z\|: z \in C\}$.

Definition 1 [10] [14]:

1) A subset $C$ is said to be proximinal if for every $x$ in $X$, the set $P_{C}(x) \neq \varnothing$.

2) A subset $C$ is said to be strongly proximinal if for any $\epsilon>0$ and any $x \in X$, there exists $\delta>0$ such that for any $y \in C$ with $\|x-y\|<d(x, C)+\delta$, then there is $y^{\prime} \in C$ with $\left\|y-y^{\prime}\right\|<\epsilon$ and $\left\|x-y^{\prime}\right\| \leq d(x, C)$. 
3) A subset $C$ is said to be uniformly proximinal if for any $\epsilon>0$ and $R>0$, there exists $\delta>0$ such that for any $x \in X, d(x, C) \leq R$ and any $y \in C$ with $\|x-y\|<R+\delta$, then there is $y^{\prime} \in C$ with $\left\|y-y^{\prime}\right\|<\epsilon$ and $\left\|x-y^{\prime}\right\| \leq R$.

From the Definition 1 , we can see uniformly proximinal $\Rightarrow$ strongly proximinal $\Rightarrow$ proximinal. For any Banach space $X$, it is easy to see $B_{X}$ is proximinal. Since for any $x \in X \backslash B_{X}, \frac{x}{\|x\|} \in P_{B_{X}}(x)$ and

$$
d\left(x, B_{X}\right)=\left\|x-\frac{x}{\|x\|}\right\|=\|x\|-1 .
$$

But, from the example by Godefroy in ([13], Pg. 87) it is clear that the closed unit ball of a Banach space not necessarily have strongly proximinal property.

Definition 2 [10] [14]: Let $X$ be a Banach space,

1) $X$ is said to be strongly ball proximinal if the unit ball $B_{X}$ is strongly proximinal.

2) $X$ is said to be uniformly ball proximinal if the unit ball $B_{X}$ is uniformly proximinal.

Definition 3 [13]: Let $X$ be a Banach space and $Y$ be subspace of $X$. We say $Y$ is an equable subspace of $X$ if for every $\epsilon>0$ there is a $\delta>0$ and a map $\psi_{\epsilon}: Y \rightarrow[0,1]$ such that for every $y \in Y,\left\|y-\psi_{\epsilon}(y) y\right\| \leq \epsilon$ and

$$
B(0,1) \cap B(y, 1+\delta) \subset B\left(\psi_{\epsilon}(y) y, 1\right) .
$$

Remark 1: In Theorem 2.6 [13], it has been proved if $Y$ is an equable subspace of $X$. Then $Y$ is strongly ball proximinal in $X$.

Let $\Psi_{\epsilon} \triangleq \psi_{\epsilon}(y) y$, we give the next lemma which is the remark 2.3 in [13] by using translation invariance of the Banach space and (2) in Definition 3.

Lemma 1 [13]: Let $Y$ be an equable subspace of a Banach space $X$, for any $\epsilon>0$, there is $\delta>0$ such that for any real scalar $r>0, y$ and $z$ in $Y$, there is $w \in Y$ with $w=r\left\{\Psi_{\epsilon}\left(\frac{z-y}{r}\right)+\frac{y}{r}\right\}$, then

$$
B(y, r) \cap B(z, r(1+\delta)) \subset B(w, r) .
$$

Additionally, if both $y$ and $z$ are in $B_{Y}$, then $w \in B_{Y}$.

Next, to avoid confusion, we use $a_{n}$ or $b_{n}$ for some real numbers, $x_{n}$ or $y_{n}$ for the vectors in Banach space.

Let $1 \leq p<\infty, l_{p}$ is the Banach space of all sequences $x=\left(a_{n}\right)_{n=1}^{\infty}$ of real so that $\|x\|_{p}=\left(\sum_{n=1}^{\infty}\left|a_{n}\right|^{p}\right)^{1 / p}<\infty$. For $p=\infty, l_{\infty}$ is the Banach space of sequences such that $\|x\|_{\infty}=\sup \left\{\left|a_{n}\right|: n \in N\right\}<\infty$.

Let $\left(X_{n}\right)$ be a sequence of Banach spaces. For $1 \leq p \leq \infty, l_{p}$-direct sum $\left(\sum \oplus X_{n}\right)_{l_{p}}$ denote the collection of elements $\left(x_{n}\right)$ such that $x_{n} \in X_{n}$ and the sequence $\left(\left\|x_{n}\right\|_{X_{n}}\right)_{n=1}^{\infty} \in l_{p}$. Thus the norm of $\left(x_{n}\right)$ is 


$$
\left\|\left(x_{n}\right)\right\|_{\left(\Sigma \oplus X_{n}\right)_{l_{p}}}=\left\|\left(\left\|x_{n}\right\|_{X_{n}}\right)\right\|_{p} .
$$

If for any $n, X_{n}=X$, we can simply denote $\left(\sum \oplus X\right)_{l_{p}}$ by $l_{p}(X)$.

\section{Main Results}

In this section, we will give our main results. For Theorem 1, we can see $B_{l_{\infty}\left(l_{2}\right)}$ is strongly ball proximinal. This result is using the "uniformly" strongly ball proximinal of the $B_{l_{2}}$ which is showed by Lemma 2. For Theorem 2, we prove when $Y$ is an equable subspace in Banach space $X, B_{Y}$ is uniformly proximinal.

Lemma 2: For every $0<\varepsilon<1 / 2$, if $x=\left(a_{n}\right)_{n=1}^{\infty} \in l_{2}$ with $d\left(x, B_{l_{2}}\right)>1 / 12$, then exist $0<\delta<\frac{\epsilon^{2}}{3}$, such that for every $y=\left(b_{n}\right)_{n=1}^{\infty} \in B_{l_{2}}$, when $\|x-y\|_{2}<d\left(x, B_{l_{2}}\right)+\delta$, we have $\left\|\frac{x}{\|x\|}-y\right\|_{2}<\epsilon$.

Proof: In this proof, we simplified the $l_{2}$ norm $\|\cdot\|_{2}$ by the symbol $\|\cdot\|$. Since $\frac{\epsilon^{2}}{3}>\delta$, so

$$
\|x\| \epsilon^{2}>3\|x\| \delta>3(\|x\|-1) \delta=2(\|x\|-1) \delta+(\|x\|-1) \delta .
$$

If $x=\left(a_{n}\right)_{n=1}^{\infty} \in l_{2}$ with $d\left(x, B_{l_{2}}\right)>1 / 12$, then by (1)

$$
\|x\|-1=d\left(x, B_{l_{2}}\right)>\frac{1}{12}>\delta,
$$

using (4) and (5),

$$
\|x\| \varepsilon^{2}>2(\|x\|-1) \delta+\delta^{2}
$$

Thus

$$
(\|x\|-1)^{2}+\|x\| \epsilon^{2}>(\|x\|-1)^{2}+2(\|x\|-1) \delta+\delta^{2}=[(\|x\|-1)+\delta]^{2} .
$$

By (5) and (6), we get

$$
\left[d\left(x, B_{l_{2}}\right)+\delta\right]^{2}=[(\|x\|-1)+\delta]^{2}<(\|x\|-1)^{2}+\|x\| \epsilon^{2} .
$$

So for any $y=\left(b_{n}\right)_{n=1}^{\infty} \in B_{l_{2}}$, when $\|x-y\|<d\left(x, B_{l_{2}}\right)+\delta$ and using (7), we have

$$
\|x-y\|^{2}<(\|x\|-1)^{2}+\|x\| \epsilon^{2},
$$

then we compute the $l_{2}$ norm by

$$
\|x\|^{2}=\sum_{n=1}^{\infty}\left|a_{n}\right|^{2}, \quad\|x-y\|^{2}=\sum_{n=1}^{\infty}\left|a_{n}-b_{n}\right|^{2},
$$

thus according to (8), we have

$$
\begin{aligned}
& \sum_{n=1}^{\infty} a_{n}^{2}-2 \sum_{n=1}^{\infty} a_{n} b_{n}+\sum_{n=1}^{\infty} b_{n}^{2}<(\|x\|-1)^{2}+\|x\| \epsilon^{2} \\
& =\|x\| \epsilon^{2}-(\|x\|-1)+\|x\|^{2}-\|x\| \\
& \leq\|x\| \epsilon^{2}-(\|x\|-1) \sum_{n=1}^{\infty} b_{n}^{2}+\sum_{n=1}^{\infty} a_{n}^{2}-\frac{\sum_{n=1}^{\infty} a_{n}^{2}}{\|x\|} .
\end{aligned}
$$


The last inequality is because $y \in B_{l_{2}}$. Then we have

$$
\frac{\sum_{n=1}^{\infty} a_{n}^{2}}{\|x\|}-2 \sum_{n=1}^{\infty} a_{n} b_{n}+\|x\| \sum_{n=1}^{\infty} b_{n}^{2}<\|x\| \epsilon^{2},
$$

which means $\left\|\frac{x}{\|x\|}-y\right\|^{2}<\epsilon^{2}$.

From the Lemma 2, let $y^{\prime}=\frac{x}{\|x\|}$, then $\left\{y^{\prime}\right\}=P_{B_{l_{2}}}(x)$ and $\left\|y-y^{\prime}\right\|<\epsilon$, this means when $x \in l_{2}$ satisfied $d\left(x, B_{l_{2}}\right)>1 / 12$, there is a "uniformly" strongly ball proximinal for these $x$. The next lemma is simple which is also needed in Theorem 1, but we give the proof for the completeness.

Lemma 3: Let $X$ be a Banach space, for $\left(x_{n}\right) \in l_{\infty}(X)$ we have

$$
d\left(\left(x_{n}\right), B_{l_{\infty}(X)}\right)=\sup \left\{d\left(x_{n}, B_{X}\right): n \in N\right\} .
$$

Proof: If $x_{n} \in B_{X}$, then $d\left(x_{n}, B_{X}\right)=0$. Thus we can assume for any $n \in N$, $d\left(x_{n}, B_{X}\right) \neq 0$.

Then $x_{n} \in X \backslash B_{X}$, so by (1)

$$
d\left(x_{n}, B_{X}\right)=\left\|x_{n}-\frac{x_{n}}{\left\|x_{n}\right\|}\right\|=\left\|x_{n}\right\|-1 .
$$

Thus

$$
\begin{aligned}
d\left(\left(x_{n}\right), B_{l_{\infty}(X)}\right) & =\inf \left\{\left\|\left(x_{n}\right)-\left(y_{n}\right)\right\|_{l_{\infty}(X)}:\left(y_{n}\right) \in B_{l_{\infty}(X)}\right\} \\
& \leq\left\|\left(x_{n}\right)-\left(\frac{x_{n}}{\left\|x_{n}\right\|}\right)\right\|_{l_{\infty}(X)}=\left\|\left(\left\|x_{n}-\frac{x_{n}}{\left\|x_{n}\right\|}\right\|\right)\right\| \\
& =\left\|\left(d\left(x_{n}, B_{X}\right)\right)\right\|_{\infty}=\sup \left\{d\left(x_{n}, B_{X}\right): n \in N\right\} .
\end{aligned}
$$

For another side, for any $\left(y_{n}\right) \in B_{l_{\infty}(X)}$, since $d\left(x_{n}, B_{X}\right) \leq\left\|x_{n}-y_{n}\right\|$, thus

$$
\left\|\left(d\left(x_{n}, B_{X}\right)\right)\right\|_{\infty} \leq\left\|\left(\left\|x_{n}-y_{n}\right\|\right)\right\|_{\infty},
$$

by the arbitrary of $\left(y_{n}\right) \in B_{l_{\infty}(X)}$, we have

$$
\sup \left\{d\left(x_{n}, B_{X}\right): n \in N\right\} \leq d\left(\left(x_{n}\right), B_{l_{\infty}(X)}\right) .
$$

Now, we can give the proof of Theorem 1.

Theorem 1: Let $X=l_{2}$, then $l_{\infty}(X)$ is strongly ball proximinal.

Proof: For every $0<\varepsilon<1 / 2$, if $\left(x_{n}\right) \in l_{\infty}(X)$ with $\left\|\left(x_{n}\right)\right\|_{l_{\infty}(X)}=r$, without loss of generality, we can assume $r=2$, thus

$$
d\left(\left(x_{n}\right), B_{l_{\infty}(X)}\right)=\sup \left\{d\left(x_{n}, B_{X}\right): n \in N\right\}=1 .
$$

Then for all $\left(y_{n}\right) \in B_{l_{\infty}(x)}$, such that

$$
\left\|\left(x_{n}\right)-\left(y_{n}\right)\right\|_{l_{\infty}(X)}=\sup \left\{\left\|x_{n}-y_{n}\right\|_{2}: n \in N\right\}<1+\frac{\delta^{2}}{8},
$$

where the $\delta$ is same as the Lemma 2. From (9) and (10), we can see for any 
$n \in N$,

$$
d\left(x_{n}, B_{X}\right) \leq 1, \quad\left\|x_{n}-y_{n}\right\|_{2}<1+\frac{\delta^{2}}{8}
$$

so we will divide into three cases to choose $y_{n}^{\prime} \in B_{X}$ so that $\left(y_{n}^{\prime}\right) \in B_{l_{\infty}(x)}$ and

$$
\left\|\left(y_{n}\right)-\left(y_{n}^{\prime}\right)\right\|_{l_{\infty}(X)} \leq \varepsilon, \quad\left\|\left(x_{n}\right)-\left(y_{n}^{\prime}\right)\right\|_{l_{\infty}(X)} \leq 1 \text {. }
$$

Case 1. $\left\|x_{n}-y_{n}\right\|_{2} \leq 1$, it is simple to choose $y_{n}^{\prime}=y_{n}$.

Case 2. $\left\|x_{n}-y_{n}\right\|_{2}>1$ and $d\left(x_{n}, B_{X}\right) \geq 1-\frac{\delta}{2}$.

Since $\delta<\frac{\epsilon^{2}}{3}<\frac{1}{12}$, so $d\left(x_{n}, B_{X}\right) \geq \frac{23}{24}>\frac{1}{12}$, then for this $x_{n} \in X$, since

$$
\left\|y_{n}-x_{n}\right\|_{2}<1+\frac{\delta^{2}}{8} \leq d\left(x_{n}, B_{X}\right)+\frac{\delta}{2}+\frac{\delta^{2}}{8}<d\left(x_{n}, B_{X}\right)+\delta \text {. }
$$

Let $y_{n}^{\prime}=\frac{x_{n}}{\left\|x_{n}\right\|_{2}}$, then by the Lemma 2

$$
\left\|y_{n}-y_{n}^{\prime}\right\|_{2}<\epsilon
$$

and we also have

$$
\left\|y_{n}^{\prime}-x_{n}\right\|_{2}=\left\|x_{n}\right\|_{2}-1=d\left(x_{n}, B_{X}\right) \leq 1 .
$$

Case 3. $\left\|x_{n}-y_{n}\right\|_{2}>1$ and $d\left(x_{n}, B_{X}\right)<1-\frac{\delta}{2}$.

Let $y_{n}^{\prime}=\left(1-\frac{\delta}{2}\right) y_{n}+\frac{\delta}{2} \frac{x_{n}}{\left\|x_{n}\right\|_{2}}$, then $y_{n}^{\prime} \in B_{X}$,

$$
\begin{aligned}
\left\|x_{n}-y_{n}^{\prime}\right\|_{2} & \leq\left(1-\frac{\delta}{2}\right)\left\|x_{n}-y_{n}\right\|_{2}+\frac{\delta}{2} \| x_{n}-\frac{x_{n}}{\left\|x_{n}\right\|_{2} \|_{2}} \\
& \leq\left(1-\frac{\delta}{2}\right)\left(1+\frac{\delta^{2}}{8}\right)+\frac{\delta}{2} d\left(x_{n}, B_{X}\right) \\
& =\left(1-\frac{\delta}{2}\right)\left(1+\frac{\delta^{2}}{8}\right)+\frac{\delta}{2}\left(1-\frac{\delta}{2}\right) \leq 1
\end{aligned}
$$

and we have

$$
\left\|y_{n}-y_{n}^{\prime}\right\|_{2}=\left\|\frac{\delta}{2} y_{n}-\frac{\delta}{2} \frac{x_{n}}{\left\|x_{n}\right\|_{2} \|_{2}}\right\|_{2} \leq \frac{\delta}{2}+\frac{\delta}{2}=\delta<\epsilon .
$$

Thus for any case, we can find the proper $\left(y_{n}^{\prime}\right) \in B_{l_{\infty}(x)}$ such that $\left(y_{n}^{\prime}\right)$ meet the requirements of (11), which means $l_{\infty}(X)$ is strongly ball proximinal.

Now we will show the uniformly ball proximinal of the equable subspace $Y$ in Banach space $X$.

Theorem 2: Let $Y$ be an equable subspace of $X$. Then $Y$ is uniformly ball proximinal in $X$.

Proof: For any $\epsilon>0$ and $R>0$ there exists $\eta=R \delta\left(\frac{\epsilon}{R}\right) \triangleq R \delta$, where $\delta\left(\frac{\epsilon}{R}\right)$ is from the equability of $Y$ which depends on $\frac{\epsilon}{R}$. Then for any $x \in X$, $d\left(x, B_{Y}\right) \leq R$. For any $y \in B_{Y}$ with $\|x-y\|<R+\eta=R(1+\delta)$, we will show 
there is $y^{\prime} \in B_{Y}$ such that

$$
\left\|x-y^{\prime}\right\| \leq R, \quad\left\|y-y^{\prime}\right\| \leq \epsilon .
$$

Note for the above fixed $x$ and $y$, there is

$$
x \in B(y, R(1+\delta)) .
$$

Since $Y$ is equable subspace of $X$, then $Y$ is strongly ball proximinal by the above Remark 1, thus $P_{B_{Y}}(x) \neq \varnothing$. So we can choose $y_{1} \in P_{B_{Y}}(x)$. Thus

$$
\left\|x-y_{1}\right\| \leq d\left(x, B_{Y}\right) \leq R .
$$

Therefore, by (13) and (14) we have

$$
x \in B\left(y_{1}, R\right) \cap B(y, R(1+\delta)) .
$$
Let $\epsilon^{\prime}=\frac{\epsilon}{R}$, then using (3) in the Lemma 1, there is $y^{\prime}=R\left\{\Psi_{\epsilon^{\prime}}\left(\frac{y-y_{1}}{R}\right)+\frac{y_{1}}{R}\right\}$
such that

$$
x \in B\left(y^{\prime}, R\right) \Rightarrow\left\|x-y^{\prime}\right\| \leq R
$$

Note, both $y$ and $y_{1}$ are in $B_{Y}$, thus $y^{\prime} \in B_{Y}$ again by Lemma 1 . Using the equability of $Y$ and Lemma 1, it is easy to see

$$
\left\|\frac{y-y^{\prime}}{R}\right\|=\left\|\frac{y-y_{1}}{R}-\Psi_{\epsilon^{\prime}}\left(\frac{y-y_{1}}{R}\right)\right\| \leq \epsilon^{\prime}
$$

thus we have

$$
\left\|y-y^{\prime}\right\| \leq R \epsilon^{\prime} \leq \epsilon .
$$

According to (15) and (16), we have found the proper $y^{\prime}$ to satisfy (12). Thus we complete the proof.

\section{Conclusion}

In this paper, we can see for these $x \in l_{2}$ with $d\left(x, B_{l_{2}}\right)>\frac{1}{12}$, they have the common $\delta$ for strong ball proximinality, then we can get the strong ball proximinality of $l_{\infty}\left(l_{2}\right)$. Also, we give an example of uniform ball proximinality. That is the equable subspace $Y$ of a Banach space $X$.

\section{Acknowledgements}

This work is supported by Huaqiao University High-level Talents Research Initiative Project (11BS220). The authors would also like to thank the Editor-in-Chief, the Associate Editor, and the anonymous reviewers for their careful reading of the manuscript and constructive comments.

\section{Conflicts of Interest}

The authors declare no conflicts of interest regarding the publication of this paper.

\section{References}

[1] Godefroy, G. and Indumathi, V. (2001) Strong Proximinality and Polyhedral Spac- 
es. Revista Matemática Complutense, 14, 105-125.

https://doi.org/10.5209/rev_REMA.2001.v14.n1.17047

[2] Bandyopadhyay, P., Li, Y., Lin, B.-L. and Naraguna, D. (2008) Proximinality in Banach Spaces. Journal of Mathematical Analysis and Applications, 341, 309-317. https://doi.org/10.1016/j.jmaa.2007.10.024

[3] Luo, X.-F., Tao, J. and Wei, M. (2019) Characterizations of Generalized Proximinal Subspaces in Real Banach Spaces. Results in Mathematics, 74, Paper No. 88. https://doi.org/10.1007/s00025-019-1013-Z

[4] Read, C.J. (2018) Banach Spaces with No Proximinal Subspaces of Codimension 2. Israel Journal of Mathematics, 223, 493-504. https://doi.org/10.1007/s11856-017-1627-3

[5] Sun, L.F. and Luo, Z.H. (2013) The Sum of Strongly Proximinal Sets. Journal of Xiamen University (Natural Science), 52, 312-315. (In Chinese)

[6] Luo, Z.H. and Sun, L.F. (2013) The Sum of Weakly Compact Sets and Proximinal Sets. Journal of Xiamen University (Natural Science), 52, 157-159. (In Chinese)

[7] Meng, Q.F., Luo, Z.H. and Shi, H.H. (2017) A Remark on the Sum of Simultaneously Proximina Subspaces. Journal of Xiamen University (Natural Science), 56, 551-554. (In Chinese)

[8] Cheng, L.X., Luo, Z.H., Zhang, W. and Zheng, B.T. (2016) On Proximinality of Convex Sets in Superspaces. Acta Mathematica Sinica (Engl. Ser.), 32, 633-642. https://doi.org/10.1007/s10114-016-5355-0

[9] Saidi, F.B. (2005) On the Proximinality of the Unit Ball of Proximinal Subspaces in Banach Spaces; a Counterexample. Proceedings of the American Mathematical Society, 133, 2697-2703. https://doi.org/10.1090/S0002-9939-05-08152-9

[10] Bandyopadhyay, P., Lin, B.-L. and Rao, T.S.S.R.K. (2007) Ball Proximinality in Banach Spaces. In: Banach Spaces and Their Applications in Analysis, Walter de Gruyter, Berlin, 251-264.

[11] Indumathi, V. and Prakash, N. (2013) Ball Proximinal and Strongly Ball Proximinal Hyperplanes. Journal of Approximation Theory, 172, 37-46.

https://doi.org/10.1016/j.jat.2013.04.006

[12] Lin, P.-K., Zhang, W. and Zheng, B. (2015) Ball Proximinal and Strongly Ball Proximinal Spaces. Journal of Convex Analysis, 22, 673-685.

[13] Lalithambigai, S. (2009) Ball Proximinality of Equable Spaces. Collectanea Mathematica, 60, 79-88. https://doi.org/10.1007/BF03191217

[14] Paul, T. (2017) Various Notions of Best Approximation Property in Spaces of Bochner Integrable Functions. Advances in Operator Theory, 2, 59-77.

[15] Lin, P.-K., Zhang, W. and Zheng, B. (2014) Stability of Ball Proximinality. Journal of Approximation Theory, 183, 72-81. https://doi.org/10.1016/j.jat.2014.04.008

[16] Godefroy, G. and Indumathi, V. (2013) Proximinality and Renormings: Some New Examples. Journal of Approximation Theory, 176, 118-128. https://doi.org/10.1016/j.jat.2013.09.004

[17] Indumathi, V. and Lalithambigai, S. (2011) Ball Proximinal Spaces. Journal of Convex Analysis, 18, 353-366. 